\title{
A Systematic Protocol Design and Management of spinal Tuberculosis by Using Antibiotics: Rifampicin, Isoniazid
}

\author{
Jashan Girdhar * \\ Department of Pharmacy Practice, Maharishi Markandeshwar deemed to be university Mullana, Ambala
} (MMCP) India.

\begin{abstract}
* Corresponding Author: Jashan Girdhar, Department of Pharmacy Practice, Maharishi Markandeshwar deemed to be university Mullana, Ambala (MMCP) India. Email: smartybmw02@ gmail.com
\end{abstract}

\begin{abstract}
Tuberculosis is commonly known as TB or Consumption it was potentially serious bacterial disease that affects the lungs basically. TB is spread when an infected person coughs or sneezes. Treatable by a medical professional It was Spreads easily, It Requires a medical diagnosis, Lab tests or imaging always required apart form that the epidemiology of Tuberculosis in India is noted on document 1 million case per year and in Madhya Pradesh, Rajasthan it is noted 75 thousands every year Patient Case Recorded. There is basically two types of tuberculosis latent TB, Active TB Respectively. In this article we are focusing on Spinal Tuberculosis who is suffering problem last 2 year vice versa. Symptoms may recorded as Chest pain, weight loss etc. The spinal column is involved in less than $1 \%$ of all cases of tuberculosis (TB). Spinal TB is a very dangerous type of skeletal TB as it can be associated with neurologic deficit due to compression of adjacent neural structures and significant spinal. In this article I explained results and justifiable conclusions as per outcome patient counselling, ADR Reports, others reports and past and Present Research/Review Article.
\end{abstract}

Keywords: Tuberculosis, Antibiotics, Spinal, Chest Pain, Neural Structure, Drug therapy.

\section{INTRODUCTION}

It is commonly known as Consumption. It can spread to other parts of your body, like your brain and spine. A type of bacteria called Mycobacterium tuberculosis causes it.In the early 20th century, TB was a leading cause of death in the United States.

Although the first documented spinal tuberculos is (TB) cases date back to 5,000-yearold Egyptian mummies, the first modern case of spinal TB was described in 1779 by Percival Pott.

Spinal involvement occurs in less than $1 \%$ of patients with TB but the increasing frequency of TB in both developed and developing countries has continued to make spinal TB a health problem [2,4]. Spinal TB (Pott's disease) is the most common as well as one of the most dangerous forms of skeletal TB and accounts for $50 \%$ of all cases of skeletal TB. Although the thoracolumbar junction seems to be the most common site of the spinal column involvement in spinal TB, any part of the spine can be affected furthermore, the incidence of neurologic complications in spinal $\mathrm{TB}$ varies from $10 \%$ to $43 \%$
Recently, the development of multidrug resistant TB, frequency of infection in immunodeficientindividuals, more accurate imaging modalities, and advances in spinal reconstruction techniques have all changed the management of Pott's disease. Advanced imaging techniques such as magnetic resonance imaging (MRI) make the early diagnosis of spinal TB easier and a considerable number of patients with spinal TB are diagnosed earlier and treated more effectively before significant neurological deficits develop. However, patients can still present late with considerable spine deformity.Since the advanced imaging modalities and different treatment protocols are developed during these years and drug resistance mandates applying innovative strategies to treat this serious disease, new reviews are always required to assess modern diagnostic moda lities and treatment options.

The objective of this article is to discuss the diagnosis and management of spinal TBaccording to the recent studies published in the literature.

Today, most cases are cured with antibiotics. But it takes a long time. Have to take meds for 
at least 6 to 9 months. In India 1 Million Cases is recorded. Tuberculosis is an infectious disease that usually affects the lungs. Compared with other diseases caused by a single infectious agent, tuberculosis is the second biggest killer, globally.In 2015, 1.8 million people died from the disease, with 10.4 million falling ill.In the 18th and 19th centuries, a tuberculos is epidemic rampaged throughout Europe and North America, before the German microbiologist Robert Koch discovered the microbial causes of tuberculos is in 1882.Following Koch's discovery, the development of vaccines and effective drug treatment led to the belief that the disease was almost defeated. Indeed, at one point, the United Nations, predicted that tuberculos is (TB) would be eliminated worldwide by 2025 . However, in the mid-1980s, TB cases began to rise worldwide, so much so, that in 1993, the World Health Organization (WHO) declared that TB was a global emergency; the first time that a disease had been labe led such as.
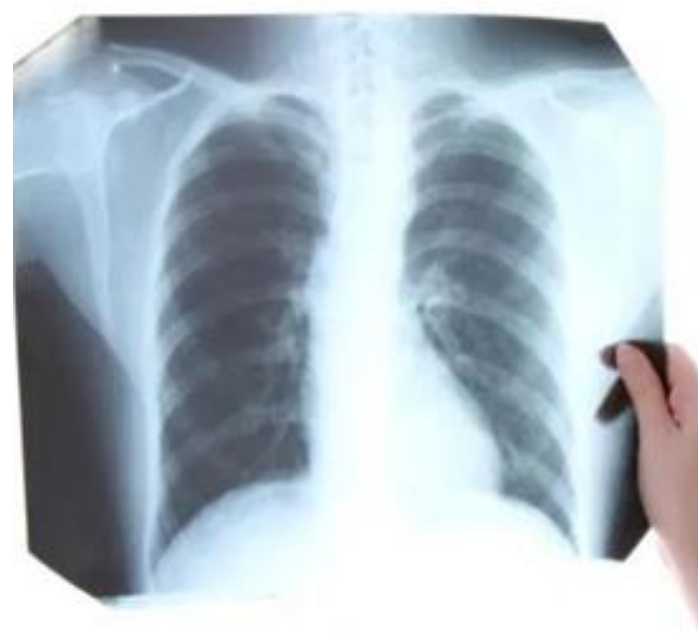

Here are some key points about tuberculosis.

The World Health Organization estimates that 9 million people a year get sick with TB, with 3 million of these "missed" by health systems

- $\quad$ TB is among the top 3 causes of death for women aged 15 to 44 .

- TB symptoms (cough, fever, night sweats, weight loss, etc.) may be mild for many months, and people ill with TB can infect up to 10-15 other people through close contact over the course of a year.

- $\mathrm{TB}$ is an airborne pathogen, meaning that the bacteria that cause TB can spread through the air from person to person

\section{TUBerCulos IS}

Tuberculosis (TB) is an infectious disease usually caused by Mycobacterium tuberculosis (MTB) bacteria. Tuberculosis generally affects the lungs, but can also affect other parts of the body.

Figure 1: TB usually affects the lungs, although it can spread to other organs around the body.

\subsection{Tuberculos is Types}

A TB infection doesn't mean get sick. There are two forms of the disease:

\subsubsection{Latent $T B$}

If the germs in body, the immune system stops them from spreading. That means don't have any symptoms and not contagious. But the infection is still alive into the body and can one day become active.treat with antibiotics to lower the risk for developing active TB.

The bacteria remain in the body in an inactive state. They cause no symptoms and are not contagious, but they can become active.

\subsubsection{Active TB.}

This means the germs multiply and can make sick. It can spread the disease to others. Ninety percent of adult cases of active TB are from the reactivation of a latent TB infection. the bacteria do cause symptoms and can be transmitted to others.About one-third of the world's population is believed to have latent TB. There is a 10 percent chance of latent TB becoming active, but this risk is much higher in people who have compromised immune systems, i.e., people living with HIV or malnutrition, or people who smoke.TB affects all age groups and all parts of the world. However, the disease mostly affects young adults and people living in developing 
countries. In 2012, 80 percent of reported TB cases occurred in just 22 countries.

\subsubsection{Early Warning Signs}

According to the Centers for Disease Control and Prevention (CDC), the symptoms of TB disease include:

Feeling sick or weak, loss of appetite and weight loss, chills, fever, and night sweats,a severe cough that lasts for 3 weeks or more,chest pain.

TB can also affect other parts of the body. Symptoms will depend on the part it affects.

\subsection{Symptoms}

During a latent stage, TB has no symptoms. When TB is active TB, the cough, fever, and other symptoms can appear.

While TB usually affects the lungs, it can also affect other parts of the body, and the symptoms will vary accordingly.

Without treatment, TB can spread to other parts of the body through the bloodstream:

- The bones: There may be spinal pain and joint destruction.

- The brain: It can lead to meningitis.

- The liver and kidneys: It can impair the waste filtration functions and lead to blood in the urine.

- The heart: It can impair the heart's ability to pump blood, resulting in cardiac tamponade, a condition that can be fatal.

\subsection{Tuberculosis Causes and how TB is Spread}

Tuberculosis is caused by bacteria that spread through the air, just like a cold or the flu. When someone who has it coughs, sneezes, talks, laughs, or sings, tiny droplets that contain the germs are released. If person breathe in these germs, person can get it.TB can spread from person to person, but it isn't easy to catch. Usually have to spend a lot of time around someone who has a lot of bacilli in their lungs.

\subsection{Tube rculos is Diagnos is}

There are two common tests for tuberculosis, but they don't tell confirmed whether you have latent or active TB:

\subsubsection{Sk in test}

This is also known as the Mantoux tuberculin skin test. A health care worker injects a small amount of fluid into the skin and lower arm.
After 2 or 3 days, they will check for swelling in arm to determine results. If results are positive, probably have been infected with TB bacteria. But the results can be false positive. If gotten tuberculosis vaccine called bacillus CalmetteGuerin (BCG), the test could say have TB when really don't. The results can also be false negative, saying that don't have TB when really do, if infection is recent. Might get this test more than once.

\subsubsection{Blood test}

These tests, also called interferon-gamma release assays or IGRAs, measure the response when TB proteins are mixed with a small amount of blood.

The most common diagnostic test for TB is a skin test where a small injection of PPD tuberculin, an extract of the TB bacterium, is made just below the inside forearm.

The injection site should be checked after 2-3 days, and, if a hard, red bump has swollen up to a specific size, then it is likely that $\mathrm{TB}$ is present. Unfortunately, the skin test is not 100 percent accurate and has been known to give incorrect positive and negative readings.

However, there are other tests that are available to diagnose TB. Blood tests, chest X-rays, and sputum tests can all be used to test for the presence of TB bacteria and may be used alongside a skin test. Multidrug-res istant TB (MDR-TB) is more difficult to diagnose than regular TB. It is also difficult to diagnose regular TB in children.

\subsection{Treatment}

The majority of TB cases can be cured when the right medication is available and administered correctly. The precise type and length of antibiotic treatment depend on a person's age, overall health, potential resistance to drugs, whether the TB is latent or active, and the location of infection (i.e., the lungs, brain, kidneys).

People with latent TB may need just one kind of TB antibiotics, whereas people with active TB (particularly MDR-TB) will often require a prescription of multiple drugs. Antibiotics are usually required to be taken for a relatively long time. The standard length of time for a course of TB antibiotics is about 6 months. TB medication can be toxic to the liver, and although side 
effects are uncommon, when they do occur, they can be quite serious. Potential side effects should be reported to a doctor and include:

Dark urine

Fever

Jaundice

Loss of appetite

Nausea and vomiting

It is important for any course of treatment to be completed fully, even if the TB symptoms have gone away. Any bacteria that have survived the treatment could become resistant to the medication that has been prescribed and could lead to developing MDR-TB in the future.Extensively drug-resistant TB (XDR TB) is resistant to some first- and second-line antibiotics. It is resistant to the most powerful TB drugs and is hard to treat. This makes it a serious risk for people with HIV and other conditions that weaken the immune system.

Directly observed therapy (DOT) may be recommended. This involves a healthcare worker administering the TB medication to ensure that the course of treatment is completed.

\subsection{Antibiotics}

Antibiotics play great role for the treatment of tuberculos is these are few categorized drugs used for treatment propose of tuberculosis are rifampic in, isoniazid.

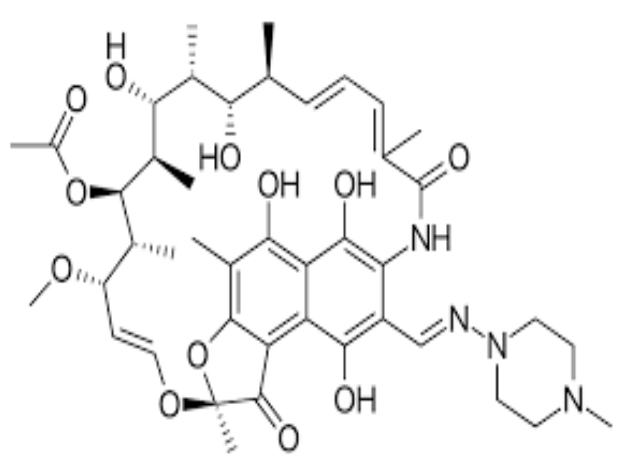

Rifampicin, also known as rifampin, is an antibiotic used to treat several types of bacterial infections, including tuberculosis, Mycobacterium avium complex, leprosy, and Legionnaires' disease.

Isoniazid is used with other medications to treat active tuberculosis (TB) infections. It is also used alone to prevent active TB infections in people who may be infected with the bacteria (people with positive TB skin test). Isoniazid is an antibiotic and works by stopping the growth of bacteria.<smiles>NNC(=O)c1ccncc1</smiles>

\section{Materials AND METHOD}

In order to extract current trends in diagnosis and medical or surgical treatment of spinal TB we performed a narrative review with analysis of all the articles available to us published between the years 1998 and 2019.

The reports about different aspects of spinal TB, with acceptable design, clearly explained results and justified conclusions according to the data were included in this review. Since, one of the aims of this article was to discuss the historical aspects of TB management we included articles regardless of their time of publication.

\subsection{Patient Information B rochure (Pib)}

Name - Mr. Jitu

Age - 33 Year

Therapy - DOT [Continuing] Since 2018 November 22

Duration of Disease - 2 Year

Weight - 57 KG [Lean Body]

Sex - Male

Location -Bhopal, India,

\subsection{Collection of Data}

Data is collected from Thombey Hospital Hyderabad, Government Medical College Indore MP.

\subsubsection{Path physiology}

There are two distinct types of spinal TB, the classic form or spondylodiscitis, and an increasingly common atypical form which is spondylitis without disc involvement. In adults, the involvement of the intervertebral disc is secondary to spread from adjacent infected vertebra.

Mr. Jitu have spondylodiscitis TB in figure 1.2, 1.3 which is shown clearly the Patient Condition in X Ray Report and CT Scan. 


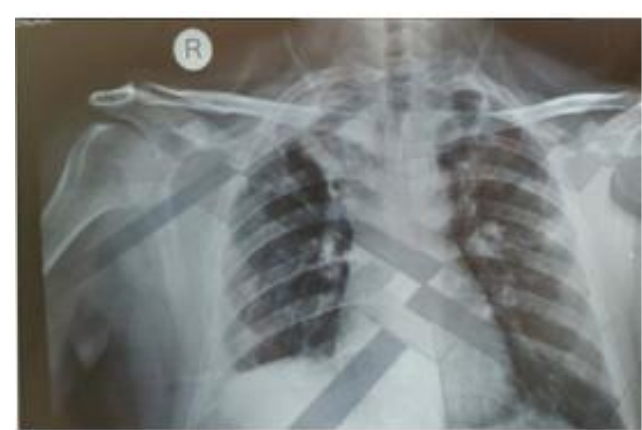

Figure2: $x$ ray Report of Mr. Jitu

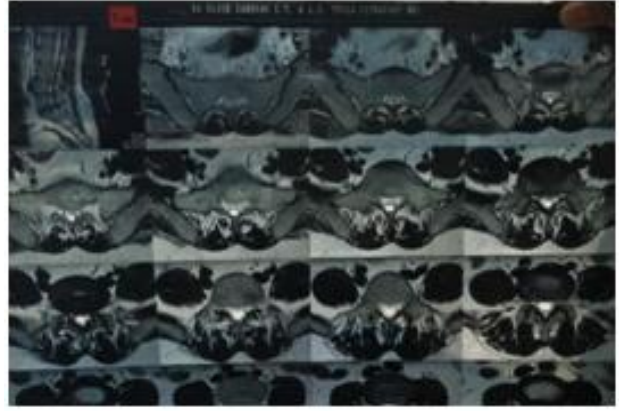

Figure3: A Patient CT Scan Report

\subsubsection{Biochemistry}

Test Name result normal Range

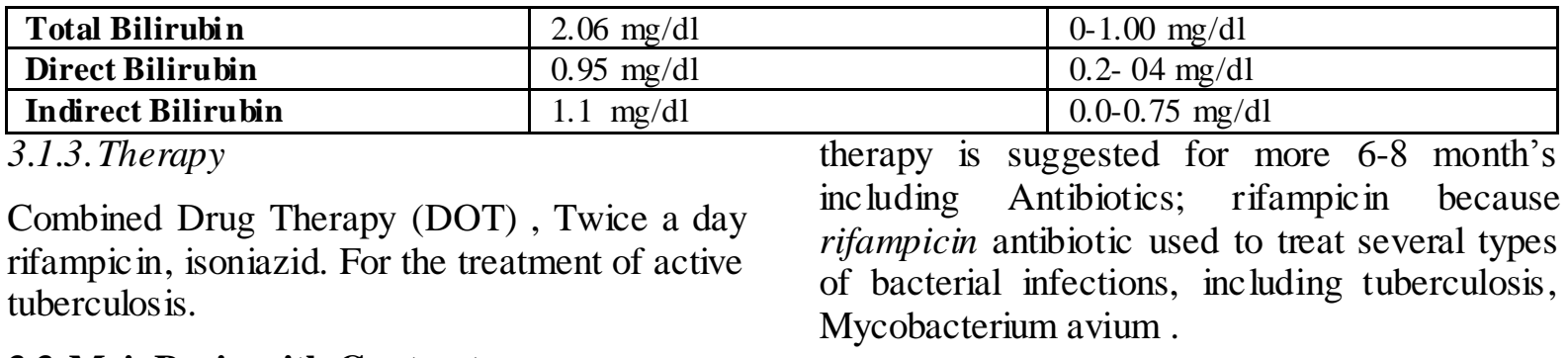

\subsection{Mri- Brain with Contrast}

\subsubsection{Techniques}

MRI of brain was performed using T1, T2, Flair, axial SWI and diffusion weight sequences in focus planes. Post Contracting Study using T1W sequences in various planes.

\subsubsection{Imaging Findings}

There is well defined ring enhancing lesion measuring approximately $12.0 \times 10.0 \mathrm{~mm}$ seen in the region of posterior limb of internal capsule on right side with mild perifocal edema. This lession is isointense to grey matter on $\mathrm{T} 1$ and T2 both. Two even smaller similar morphology lessions seen in cortical / extra axial region of left frontal lobe. Similar lesions are also seen in left basifrontal region and bilateral regions.

\section{RESULTS AND DisCUSSION}

In a Patient of pulmonary tuberculosis presented with seizures, above MRI Findings of Multiple ring enhancing lessions with perifocal edema suggest possibility of tuberculosis.

\section{CONCLUSion}

Spinal tuberculosis is very serious disorder overall Discussion and as Per X Ray, MRI, CT Scan Reports, and overall findings of patient edema suggested possibility of tuberculosis. $\mathrm{Mr}$. Jitu is suffering a series problem such as pain in spinal cord and Neuro disturbance. The DOT

\section{ACKNOWLEDGEMENT}

First of all I would like to Thank Ms. Umama Yezdani (Department of Clinical and Pharmacy Practice) Intern Thambey Hospital Hyderabad, India. I also thankful to Md Gayoor khan for proper guidance and encouragement, also thankful to Dr.Sumeet gupta, PhD. Principal MMDU Department of Pharmacy Practice, Dr Parminder Nain, Dr inderjeet Verma, for ceaseless encouragement during the study and for full support.

\section{REFERENCES}

[1] Mohammad Gayoor Khan, Umama Yezdani, Shivam Choudghal, Sana Rahman, Mayur Sadar, Ayush Kumaret.al. A Brief Study on Motor Neuron Disease; and its Treatment Includes Drug Delivery System; Future Aspects on Neurological Science" IOSR Journal of Pharmacy and Biological Sciences (IOSRJPBS) 14.4 (2019): 52-56.DOI:10.9790/30081404015256

[2] UmamaYezdani, Mohammad Gayoor khan, ZubiaZainab, Mayur sadar, Shivam Choudghal, Damini Mishra, Hari Baska. The Current Scenario of Pharmaceutical Research; 3d Tissue Engineering Considered as Eminent Technique. ARC Journal of Public Health and Community Medicine.2019; 4(2):16-22.

[3] Gayoor Khan, Umama Yezdani, Rohit Verma, Raqshan Jabeen, Pradeep Sintha. Detection of Phlebovirus by using qualitative Real time (RT) - PCR and application of silver nanoparticles to 
control it. World J Pharm Pharm Sci.2018; 7(11):936-52.

[4] Mohd. Gayoor Khan. The Novel Drug Delivery System. World J Pharm Pharm Sci. 2017; 6(7): 477-487.

[5] Unama Yezdani, Mohd. Gayoor Khan, Fazal Khan, Arvind Verma, Nilesh Kushwah, Rohit Verma.The Drug Targeting in Alzheimer's or Applications \& it's Hazards. World J Pharm Sci.2017; 7(11):1532-1549.

[6] Dr.H. S Chandel., Sharad P. Panday, Arvind Dangi, Ashish chaurasia, Mohd.Gayoor khan et al. Development of Targeted Drug delivery. International Journal of research methodology Ijrm.Hu man. 2017 Vol. 1 (2): 30-34.

[7] Kushwah Nilesh, Yezdani Umama, Mohammad Gayoor khan, Manish kushwah, Kumar Ayush.The Fundamental of Novel Drug Delivery System; Methodology, Role of Nanotechnology; Nanoparticles in Pharmaceutical Research. International Journal of Emerging Technologies and Innovative Research (www.jetir.org), ISSN: 2349-5162, Vol.6, Issue 6, page no.140-146, June-2019. DOI: http://doi.one/10.1729/Journal.21510

[8] Mohd. Gayoor Khan, Nilesh Kushwaha, Fazal Khan, Vipul Patel. Microencapsulation. International Journal of research methodology Ijrm. Hu man. 2017 Vol. 1 (2):35-42.
[9] Sai Kiran NA, Vaishya S, Kale SS, Sharma BS, Mahapatra AK. Surgical results in patients with tuberculosis of the spine and severe lowerextremity motor deficits: a retrospective study of 48 patients. J Neurosurg Spine. 2007; 6:320326. [PubMed] [Google Scholar]

[10] Rezai AR, Lee M, Cooper PR, Errico TJ, Koslow M. Modern management of spinal tuberculosis. Neurosurgery. 1995; 36:87-97. [PubMed] [Google Scholar]

[11] Turgut M. Spinal tuberculosis (Pott's disease): its clinical presentation, surgical management, and outcome. A survey study on 694 patients. Neurosurg Rev. 2001;24:8-13. [PubMed] [Google Scholar]

[12] Barnes PF, Bloch AB, Davidson PT, Snider DE., Jr Tuberculosis in patients with human immunodeficiency virus infection. $N$ Engl J Med. 1991; 324:1644-1650. [PubMed] [Google Scholar]

[13] Moorthy S, Prabhu NK. Spectrum of MR imaging findings in spinal tuberculosis. AJR Am J Roentgenol. 2002; 179:979-983. [PubMed] [Google Scholar]

[14] Pertuiset E, Beaudreuil J, Liote F, et al. Spinal tuberculosis in adults, A study of 103 cases in a developed country, 1980-1994. Medicine (Baltimo re) 1999; 78:309-320. [PubMed] [Google Scholar].

Citation: Jashan Girdhar, A Systematic Protocol Design and Management of spinal Tuberculosis by Using Antibiotics: Rifampicin, Isoniazid. ARC Journal of Public Health and Community Medicine. 2019; 4(4):2328. DOI: dx.doi.org/10.20431/2456-0596.0404004.

Copyright: (c) 2019 Authors. This is an open-access article distributed under the terms of the Creative Commons Attribution License, which permits unrestricted use, distribution, and reproduction in any medium, provided the original author and source are credited. 